\title{
VARIA
}

\section{EL RETRATO DEL OBISPO JAN MALDERUS DE VAN DYCK IDENTIFICADO EN LA ANTIGUA COLECCIÓN DEL MARQUÉS DEL CARPIO}

No es fácil exponer el estado de la cuestión en cuanto a los retratos conocidos del obispo Jan Malderus, con diferente grado de calidad y copiosa documentación literaria. Los últimos estudios reclaman la existencia de un original perdido, al tratar de la repetición de taller de la antigua colección de Pierre Crozat en París [Lienzo, $115 \times 96 \mathrm{~cm}{ }^{1}$. Hoy pienso que se trata de la tabla que se encuentra en la actualidad en la Fundación BSCH, desconocida en los estudios consagrados al pintor $[125 \times 97 \mathrm{~cm} \text {. Fig. 1 }]^{2}$.

Los grabados de Johannes Meysens [Fig. 3] ${ }^{3}$ y Adrianus Lommelin [Fig. 4] ${ }^{4}$, testifican la identidad del personaje, y el encargo a Van Dyck por parte del omnipotente obispo. El último no está invertido respecto a la pintura como sería habitual, pero es coherente con la tabla que estudiamos y con las repeticiones que tratamos a continuación.

Los dos ejemplares de mayor calidad hasta ahora localizados, el de la antigua colección Crozat antes mencionado [Fig. 2] y el del Museo Real de Amberes [Fig. 5] ${ }^{5}$, no han conven-

\footnotetext{
${ }^{1}$ Barnes, S. J.; De Porter, N.; Millar, O.; Vey, H., Van Dyck. A Complete Catalogue of the Paintings, Yale, New Haven - Londres, 2004, n. ${ }^{\circ}$ III. A17, p. 407.

${ }^{2} \mathrm{Di}$ a conocer este retrato en la Exposición Rubens y su Siglo, Méjico, Museo Nacional de San Carlos, 1998, p. 201, $\mathrm{n}^{\circ}$ 88. En el reverso existe un sello de lacre con escudo de armas, de interés estudairlo en el campo de la heráldica para su identificación.

${ }^{3}$ «PERILL. [ris] et REVEREN: [mus] DOMINVS IOANNES MALDERVS/ EPISCOPUS ANTVERPIENSIS// Antonius van Dyck pinxit/ WHollar fecit aqua forti, Antwerpiae, A [o] 1645/ Ioannes Meysens excudit». [Mauqouy-Hendrickx, L'iconographie d'Antoine Jan van Dyck, Catalogue raisonné, Académie Royale de Belgique, Classe des Beaux-Arts, 9, 2 vols., Bruselas, 1956, p. 293, n. ${ }^{\circ} 133$; The New Hollstein. Dutch \& Flemish Etchings, Engravings and Woodcuts 14501700, Anthony van Dyck, Rotterdam, 2002, III, pp. 124-125, n..$\left.^{\circ} 147\right]$.

${ }^{4}$ Contiene la inscripción: «PELL [ris] et REVEREN [mus] DOMINVS D. IOANNES MALDERVS. Y abajo a la izquierda: «Ant. Van Dÿck pinxit»; y a la derecha «Adrianus Lommelin sculpsit» [Mauqouy-Hendrickx, Op. cit. 1956, p. 339, n. ${ }^{\circ}$ 178; New Hollstein, Op. cit., 2002, pp. 126-127, n. ${ }^{\circ} 148$.

${ }^{5}$ Tabla, $117 \times 94 \mathrm{~cm}$. Amberes, Koninklijk Museum voor Schone Kunsten [Cat. Amberes 1958, n. ${ }^{\circ}$ 402; Cat. 1970, p. 70; Koninilijk Museum voor Schone Kunsten 1988, p. 115]; Smith, J., A Catalogue Raisonné of the Works of the Most Eminent Dutch, Flemish and French Painters, III, Londres, 1831, n. ${ }^{\circ} 20$; Waagen, G.F., Die Gemäldesammlung in der kaiserlichen Ermitage zu St Petersburg, Munich 1864, p. 153; Guiffrey, J., Anthonie van Dyck et son oeuvre, París, 1882, $\mathrm{n}^{\circ}$ 667; Cust, L., Anthony van Dyck: An Historical Study of His Life and Works, Londres, 1900, p. 257, n. ${ }^{\circ}$ 67; Rooses, M., Vijftig meesterwerken van Antoon van Dyck, Amberes, 1900, pp. 24-25; Shaëffer, E., Van Dyck. Des Meisters Gemälde, Klassiker der Kunst, Stuttgart-Leipzig, 1909, p. 505, il. 226; Glück, G., Van Dyck. Des Meisters Gemälde, Stuttgart-Berlín, 1931, p. 298, nota en p. 551 (Cita copias de Erasmus Quellinus y otras); Glück, G., Rubens, Van Dyck und ihr Kreis, Viena, 1933, pp. 168, 390; Van den Wijngaert, F., Antoon van Dyck, Amberes, 1943, p. 97; Van den Wijngaert, F., Catalogus Van Dyck Tentoonstelling, Koninklijk Museum Antwerpen, 1949, p. 28, n. ${ }^{\circ} 32$; Cat. Spring Collection, Frank T. Sabin Gallery, Londres, 1952, no 28; 100 Opere di Van Dyck, Cat. Exp. Génova, Palazzo de la Academia, 1955, p. 41, n. ${ }^{\circ}$ 85; Mauqouy-Hendrickx, Op. Cit. 1956, n. ${ }^{\circ} 133,178$; Larsen, E., The Paintings of Anthony van Dyck, Freren, 1988, II, pp. 221-222, n. ${ }^{\circ}$ 549; Barnes-Millar-Porter-Vey, Op. cit. 2004, p. 407, in n. ${ }^{\circ}$ III.A.17.
} 
cido en la última monografía consagrada a Van Dyck ${ }^{6}$. Ambos eran catalogados como auténticos hasta fechas recientes. La referencia más antigua del ejemplar del Museo de Amberes data de 1812, en que se localiza en el antiguo Palacio Obispal de Amberes. De la procedencia del primero, en manos de la familia Crozat hasta 1770, se sabe pasó a la propiedad de Catalina II de Rusia y al Ermitage de San Petersburgo, saliendo al mercado artístico de Londres el 25 de noviembre de $1960^{7}$. Desde entonces se venía ignorando su paradero hasta el año 2005, en que consta en el comercio neoyorquino, con atribución a Van Dyck y taller. Se incluye en el catálogo de la galería neoyorquina la opinión del profesor Vey, que reconoce su calidad y la mano de Van Dyck en el rostro, y colaboración del taller en el hábito del retratado. Vey se decanta por un criterio más positivo que lo expuesto en la monografía de 2004, juzgando a través de fotografías en color y transparencias ${ }^{8}$. Esta pintura cuenta con abundantes referencias literarias en los biógrafos de Van Dyck ${ }^{9}$, con distintas opiniones en cuanto a su autoría precisa. A mediados del siglo xx, se tuvo por el original, atribuyendo el ejemplar del Museo de Amberes a Erasmus Quellinus ${ }^{10}$. Nosotros reconocemos en esta obra una notable calidad, de acuerdo con el profesor Vey. Es difícil desligar la participación directa de Van Dyck en alguna medida.

El retrato del obispo objeto de este estudio procede de la casa real portuguesa de Braganza. Con atribución a Rubens pasó al coleccionismo privado de Madrid y a la fundación donde se encuentra en la actualidad. El estudio de la pintura me proporcionó argumentos para reconocer una calidad superior a las repeticiones y copias conocidas. Se expuso en fechas recientes en el Museo de San Carlos de México, restituyéndola a Van Dyck, en espera de un estudio más detallado ${ }^{11}$.

Ninguna noticia conocíamos hasta ahora sobre la procedencia de esta pintura en el coleccionismo de Portugal, pero es importante para su historia externa reconocer la proximidad dinástica de Portugal y España en el siglo xvir. La calidad y el análisis estilístico son el apoyo decisivo para fundamentar su vinculación con Van Dyck, a pesar de la atribución tradicional a Rubens y el prestigio de su origen inmediato.

Una reciente noticia documental en la última monografía de Van Dyck (2004), viene a potenciar nuestra tesis sobre este retrato desconocido de Malderus, que encuentro vinculado al que consta adquirido por el conde de Fuensaldaña, con destino a la Corte española, en $1651^{12}$, formando parte de unos lotes que gestiona en Londres con ocasión del derrocamiento de Carlos I de Inglaterra. Este lote se destinaba a la Colección Real y a otras colecciones de nobles españoles, como el Marqués del Carpio, ávido por la adquisición de las colecciones inglesas puestas entonces en venta. Esto es un hecho bien conocido. La partida a la que

\footnotetext{
${ }^{6}$ Barnes-Millar-Porter-Vey, Op. cit., 2004, p. 407.

${ }^{7}$ Londres, Christie's, 25 de noviembre de 1960, n. ${ }^{\circ} 69$.

${ }^{8}$ Sotheby's, Nueva York, 27-01-2005, n. ${ }^{\circ} 139$.

9 J. B. de la Curne de Sainte-Pelaye, Catalogue des Tableaux du Cabinet de M. Crozat, Baron de Thiers, París, 1755 , pp. 43-44 ; La Galerie Impériale..., San Petersburgo, 1838, p. 434, n. ${ }^{\circ} 13$; Guiffrey, Op. cit. 1882, p. 270, n. $677 \mathrm{~b}$; Rooses, Fifty Masterpieces of Anthony Van Dyck in Photogravure Selected from the Pictures Exhibited at Antwerp in 1899, traducción de F. Knowles, Londres, 1990, p. 25; Cust, Op. cit. 1900, p. 257, in n. ${ }^{\circ}$ 67; A. Somof, Ermitage Imperial. Catalogue de la Galerie des Tableaux, II, San Petersburgo, 1901, p. 87, n. ${ }^{\circ} 638$; Shaëffer, Op. Cit. 1909, p. 505, n. ${ }^{\circ} 226$; Glück, Op. Cit. 1931, n. ${ }^{\circ} 13$, 2. $^{\mathrm{a}}$ ed. p. 551, n. ${ }^{\circ}$ 248; E. Dacier, Gabriel de Saint-Aubin, II, París-Bruselas, 1931, pp. 1901, n. ${ }^{\circ}$ 1030; Spring Exhibition, Frank T. Sabin, Londres, 1952, n. ${ }^{\circ} 28$; Barnes-Millar-Porter-Vey, Op. cit. 2004, p. 417 , n. ${ }^{\circ}$ III.A17.

${ }^{10}$ Spring Exhibition, Frank T. Sabine, Londres, 1952, n. ${ }^{\circ}$ 28; Barnes-Millar-Porter-Vey, Op. cit. 2004, p. 407 , n. ${ }^{\circ}$ III.A17. En la última exposición de Amberes de 1999 ya se indica la calidad superior de la versión procedente de la antigua colección del Ermitage. [Baisier, C. - Mannaerts, R. - Wolters, B. - Van der Wey], Antoon Van Dyck anders bekeken, Over registers en contrefeytsels, tronies en copyen in Anterpse Kerken en Kloosters, Onze-Lieve-Vrouwekathedraal, Amberes, 1999, n. ${ }^{\circ}$ ]

${ }^{11}$ Díaz Padrón, M. y Padrón Mérida, A., Rubens y su siglo, Cat. Exp. Mexico, Museo Nacional de San Carlos, 1998, p. 211, n. $^{\circ} 88$.

12 Barnes-Millar-Porter-Vey, Op. cit. 2004, p. 407.
}

$A E A$, LXXVIII, 2005, 312, pp. 413 a 438 


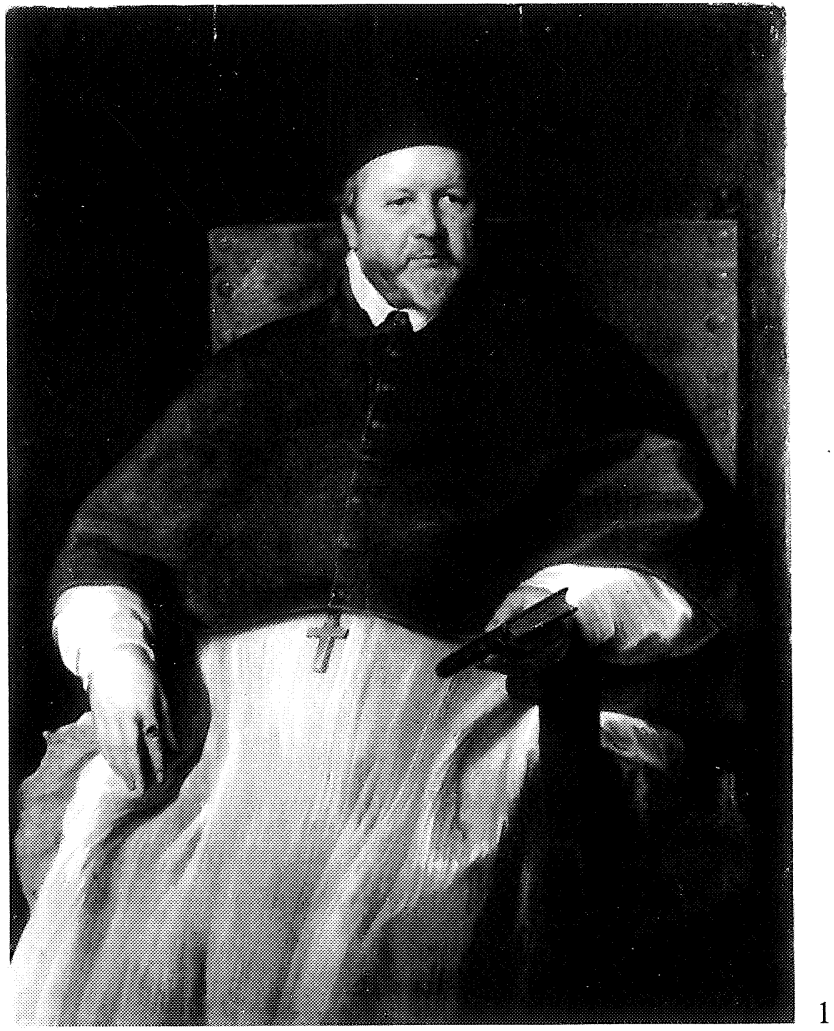

2

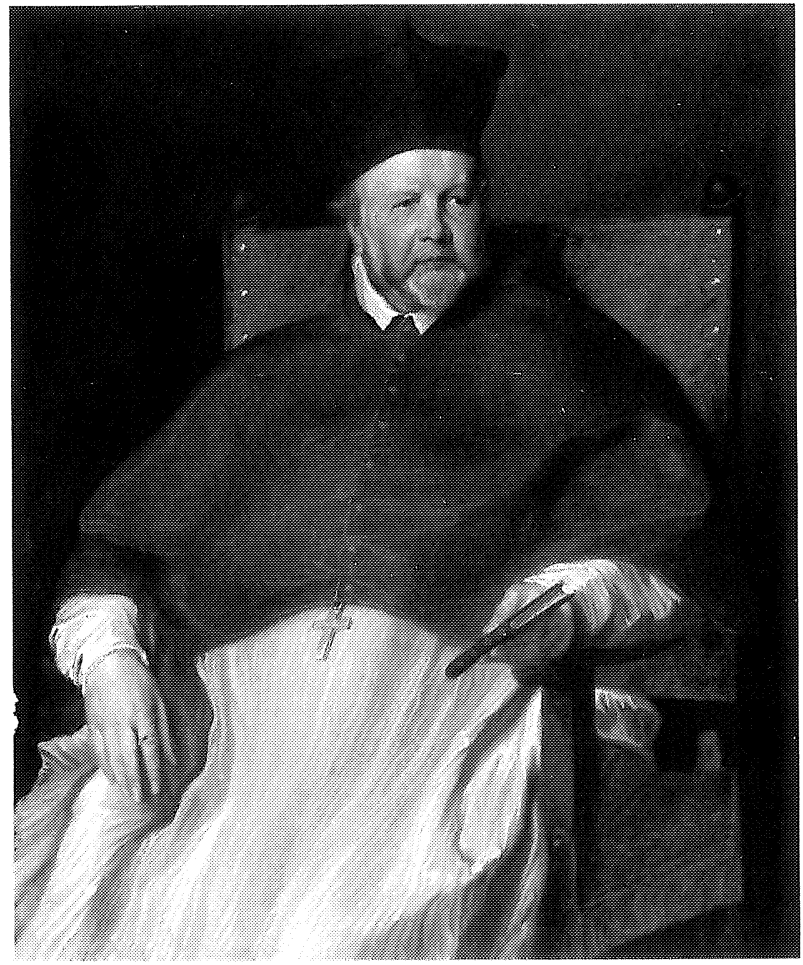

Fig. 1. Anton van Dyck. Retrato de Jan Malderus. Madrid, Fundación BSCH.

Fig. 2. Anton van Dyck y taller. Retrato de Jan Malderus. Antigua colección Crozat.

AEA, LXXVIII, 2005, 312, pp. 413 a 438 
nos referimos dice lo siguiente: «un retrato de obispo sentado en una silla con las manos sobre la misma silla de Antonio van Dyck» ${ }^{13}$. En la última monografía de Van Dyck esta referencia se asocia a un retrato de Malderus sin localizar, reconociendo la garantía de autenticidad del documento, pasando revista a las conocidas. Pensamos que la pintura fue retenida por don Gaspar de Haro y Guzmán, Marqués del Carpio y Eliche, en cuyo inventario de 1689 se registra el retrato, en el aposento detrás del jardín de San Joaquín: «210 : «Un Rettratto de Un obpo de medio Cuerpo original de Vandique de Vara y media de Caida y Vara y terzia de ancho Con marco negro en mill y quinienttos Rs. 1 . 500» ${ }^{14}$. La traducción de varas a centímetros encaja bien con las medidas actuales y permite pensar que se trata de la misma pintura. Hoy reconocemos el original que se ha tenido por perdido; localizado en un medio tangencial a la corte española sin salir de la península ibérica en los nexos de la familia real y nobleza.

Este retrato de Malderus debió impactar por las numerosas repeticiones, copias y grabados que, en parte hemos citado. El prestigio de este obispo fue el motivo de la divulgación de su imagen en el ámbito hispanófilo de los Países Bajos. Una copia hizo Erasmus Quellinus según Van Dyck ${ }^{15}$. Otra en la colección de Jeremías Wildens en $1650^{16}$ y otra en colección privada de Viena, que Glück atribuyó a Jordaens [Fig. 6] ${ }^{17}$.

Con indumentaria de obispo, cuerpo de formas llenas y pesadas y vigoroso continente, Malderus está sentado de frente al espectador, con el rostro vuelto ligeramente hacia la derecha, aunque concentrado en sus pensamientos. Un sutil ritmo divergente se dibuja en las rodillas y en el brazo apoyado en la silla, fijando una perspectiva hacia la izquierda. Es un efecto que viene acentuado por el brazo y la mano con el libro, en un difícil escorzo. Van Dyck compensa y rompe la verticalidad, sin por ello perder la frontalidad dominante de su imagen. Logra la monumentalidad y contención expresiva que puso en el Retrato de Martin Ryckaert del Museo del Prado. Van Dyck logra transmitir la personalidad del prelado con acierto y potencial sobriedad, renunciando a cualquier halago. La mirada profunda y estrábica del Obispo busca la trascendencia de la vida en su dimensión teológica. El rostro y las manos gruesas, la expresión bondadosa y la mirada penetrante y viva dominan sin paliativos la composición. Van Dyck retoma la fórmula aplicada a los hombres notables de la Iglesia y de la ciencia, como Rubens en los retratos de Van Thulden y Ofovius. Van Dyck combina la variedad de ritmos con la prestancia frontal.

La composición deriva en última instancia de fórmulas tizianescas, que Van Dyck asume de forma directa y a través de Rubens. La monumentalidad está más acorde con las interpretaciones rubenianas, lo que justifica la antigua atribución al maestro en la colección de los Braganza. Recuerda la fórmula de Tiziano en el Retrato de Francisco de Sajonia en la Pinacoteca de Viena. La poderosa plasticidad, el sólido colorido y las trasparencias de tradición nórdica son motivo para su enclave en el segundo periodo de Amberes (1626-1632).

El retrato del Museo de Amberes se ha vinculado con un retrato de Erasmus Quellinus inventariado a finales del siglo XVII ${ }^{18}$. Se documentan obras de medio cuerpo con libros detrás y

\footnotetext{
${ }^{13}$ Vergara, A., «The Count of Fuensaldaña and David Teniers: their purchases in London after the civil war», The Burlington Magazine, 1989, p. 130, n. ${ }^{\circ}$ 13; Barnes-Millar-Porter-Vey, Op. cit. 2004, p. 407. Sin asociar al retrato de Malderus que vinculamos en estas páginas.

${ }^{14}$ Cit. Burke, P. \& Cherry, P., Spanish Inventories, Collections of Paintings in Madrid, 1602-1755, Getty Information Institute, 1997, p. 842, n. ${ }^{\circ} 207$, donde se desconoce su identificación con Malderus.

15 «Malderus van Erasmus Quellinus naer Van Dyck» [Denucé, J., De Antwerpsche «Konstka mers» Inventarissen van Kunstverzamelingen te Antwerpen in de 16e en 17. Amberes, 1932, p. 283].

16 «Een contrefeytsel van den Bisschoop Johannes Malderus, naer Van Dyck» [Denucé, Op. cit. 1932, p. 164]

${ }_{17}$ En 1930 en la colección de Adela Fischel (Drei Jahrhunderte vlämischer Kunst, Viena, 1919).

${ }^{18}$ Duverger, E., Antwerpse kunstinventarissen uit de zeventiende eeuw, Bronnen voor de Kunstgeschiedenis van de Nederlanden, Bruselas, X, p. 656, y otra copia de 1653, Ibidem, VI, p. 489.
}

AEA, LXXVIII, 2005, 312, pp. 413 a 438 

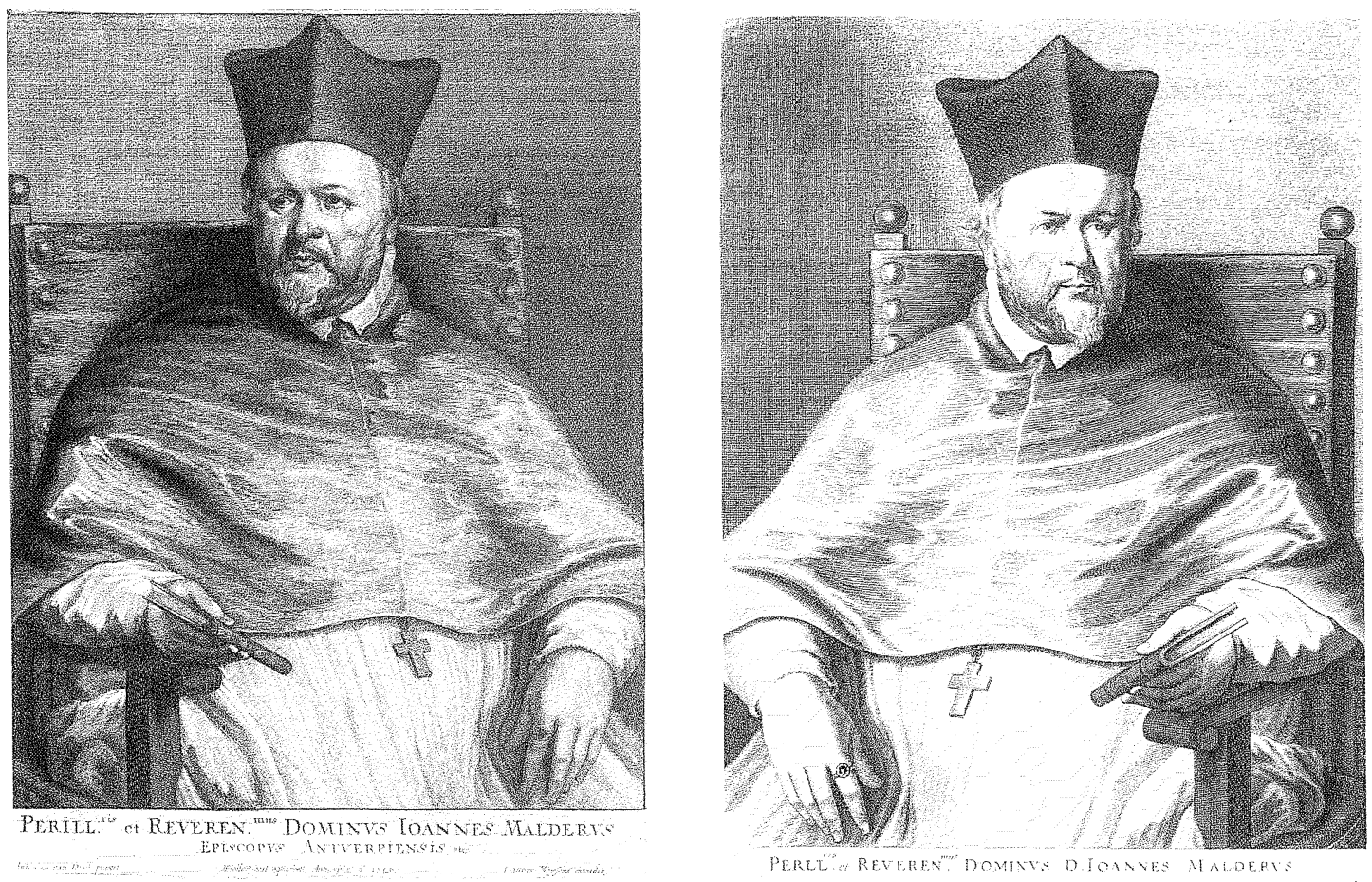

5
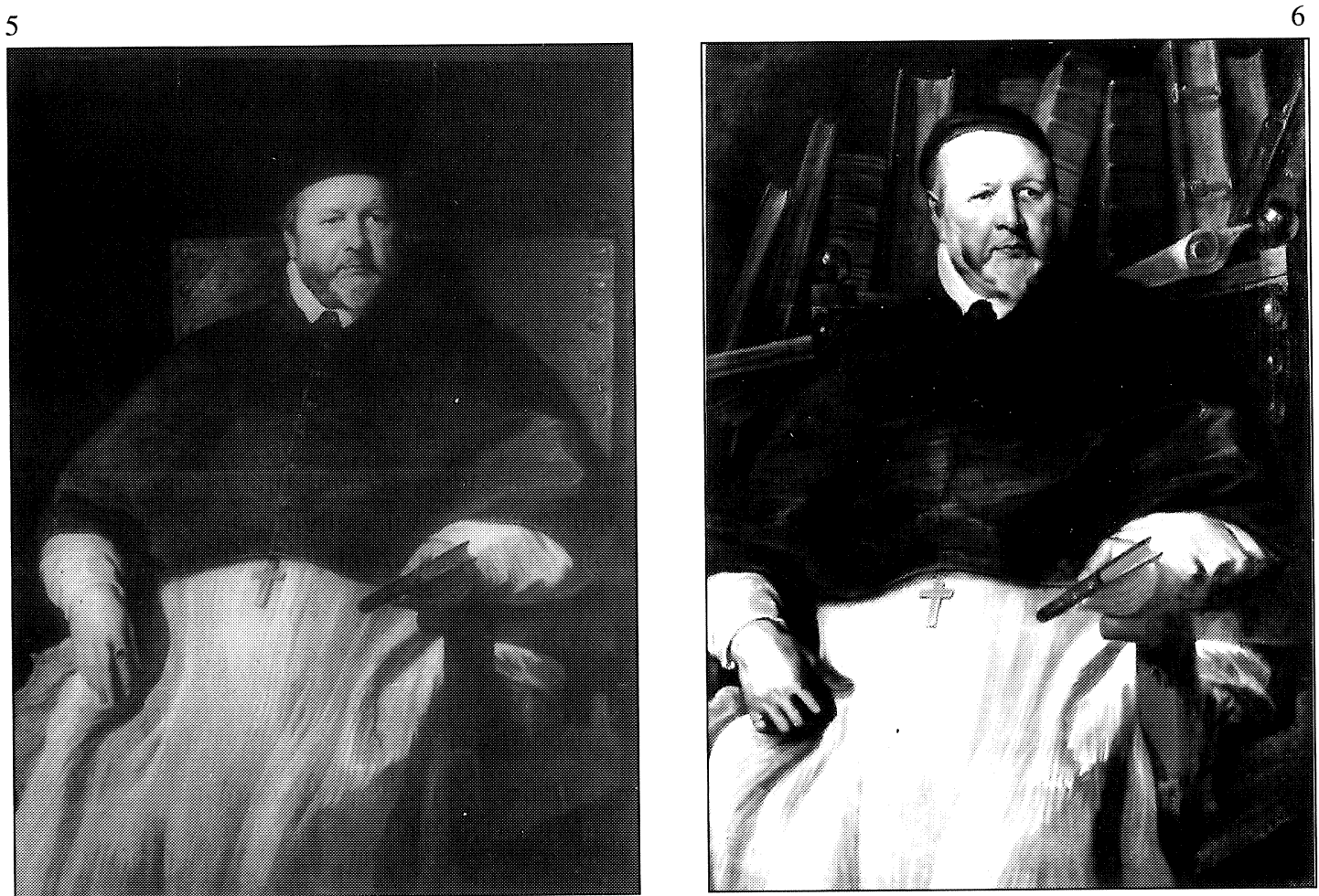

Fig. 3. Johannes Meysens según Van Dyck. Retrato de Jan Malderus. Grabado.

Fig. 4. Adrianus Lommelin según Van Dyck. Retrato de Jan Malderus. Grabado.

Fig. 5. Anton van Dyck Taller. Retrato de Jan Malderus. Amberes, Koninklijk Museum voor Schone Kunsten.

Fig. 6. Copia según Anton van Dyck (atribuida a J. Jordaens). Retrato de Jan Malderus. Viena. Colección privada.

AEA, LXXVIII, 2005, 312, pp. 413 a 438 
la mano derecha cerrada respecto al original de Van Dyck, atribuidas a Jordaens en el mercado artístico de Viena. Otras más, de busto, han aparecido en Amberes (Openbard), Dorotheum, $1979^{19}$, en el museo de la ciudad de Los Ángeles, Galería real del Buckingham Palace, en venta de Berlín de $1913^{20}$, y en el mismo lugar en $1916^{21}$. Otros en el Palacio de Bellas Artes de la Comuna de París, Museo de Versalles e iglesia de Nuestra Señora de Amberes.

Jan Malderus (Leeuw-Saint-Pierre, 1536 - Amberes, 1633) fue obispo de Amberes, teólogo destacado y fundador del Colegio de Teología anejo a la Universidad de Lovaina. Educado bajo la tutela de su tío paterno, Jan van Malderen, estudió Humanidades en Bruselas, Filosofía en la Universidad de Douai y Teología en Lovaina. Obtuvo la cátedra de Filosofía en dicha universidad y en 1594 fue nombrado Doctor en Teología. En 1596 fue solicitado por Felipe II para ocupar la cátedra de teología escolástica de Santo Tomás, y la presidencia del Seminario pastoral destinado a la instrucción de eclesiásticos en Lovaina. En 1602 fue nombrado rector de la Universidad. Desempeñó estos cargos con tal éxito, que en 1611 fue llamado por los archiduques Alberto e Isabel Clara Eugenia para ocupar el obispado de Amberes. Intervino en el embellecimiento de las iglesias de su diócesis, en las que colocó a sacerdotes instruidos. Fue hombre piadoso y culto. Practicó numerosas donaciones a las instituciones religiosas. Son numerosas sus obras escritas sobre la Summa teológica y Tratados del sueño de Santo Tomás, así como sobre la Santísima Trinidad y numerosas reflexiones filosóficas inclinadas a la moral práctica ${ }^{22}$.

Matías Díaz PADRón

\footnotetext{
${ }^{19}$ Dorotheum, 24 de abril de 1979, n. ${ }^{\circ} 53$.

${ }^{20}$ Berlín, Lepke, 14-15 de octubre de 1913, n. ${ }^{\circ} 80$.

${ }^{21}$ Berlín, Lepke, 8 de febrero de 1916, n. ${ }^{\circ} 128$.

${ }^{22}$ Geestelyck onerwys, tot versterking van den crancken in 't geloove, Amberes, 1613; De virtibus theologicis, et justicia et religione, comentaria ad $2 a m 2^{a} D$. Thomae, Amberes, 1616; Modus procedendi in curia eclesiástica, Amberes, 1619; Anti-synodica, sive animadversiones in decreta conventos Dordraceni, quam vocant synodum nationalem, de quinque doctrinae capitibus, inter remonstrantes et contraremonstrantes controversis, Amberes, 1620 (obra de gran repercusión, incluso para los protestantes) ; Tractatus de sigillo confessionis sacramentalis, Amberes, 1626 ; Comentarii de $S$. Trinitate, Creatione in genere, et de Angelis, ad I partem D. Thomae, Amberes, 1634; [Vid Ch. Piot, en Biographie Nationale Publiée par l'Académie Royale des Sciences, des Lettres et des Beaux-Arts de Belgique, vol. XIII, p. 223]; Clercq, C. de, Het bisdom Antwerpen 1559-1962, Amberes, 1962, p. 18; Aerts, W., De Onze-Lieve-Vrouwekathedraal in Antwerpen, Amberes, 1993, p. 20; Grieten, S., De Onze-Lieve-Vrouwerkathedraal van Antwerpen: Kunstpatrimonium van het Ancien Régime, Brepols, Turnhout, 1996, p. 11, 124, 432-437, n. ${ }^{\circ} 991$.
}

AEA, LXXVIII, 2005, 312, pp. 413 a 438 